\title{
Leptomeningeal carcinomatosis in a patient with metastatic pancreatic cancer: An unusual case presentation
}

Jimenez Duque D, Garcia Escobar I*, Ferragut Lloret F, Portela Gutierrez J, Gonzalez Casares S, Pineda Pineda MD, Villa Guzman JC, Cano Cano JM and Espinosa Arranz J

Medical Oncology Department, General Universitary Hospital of Ciudad Real, Spain

\begin{abstract}
Leptomeningeal carcinomatosis is characterized by multifocal seeding of the meninges by malignant tumor cells. It is a rare and generally manifests in advanced stage of solid tumors, being most common in breast and lung cancer. In the pancreatic cancer, the spread to the leptomeninges is extremely rare, although it can become an increasingly common affectation if the overall survival of these patients increases. The clinical presentation is non-specific, but usually neurological symptoms, and diagnosis includes MRI and cerebrospinal fluid cytology. Below, we present a case of a patient diagnosed with pancreatic adenocarcinoma of more than one year's evolution who develops meningeal carcinomatosis.
\end{abstract}

\section{Introduction}

Pancreatic cancer has a high mortality rate as less than $20 \%$ of patients are amenable to surgical treatment at the time of diagnosis [1]. In metastatic disease, overall survival at 5 years is less than $5 \%$. The organs where metastasis occurs most frequently are the liver and peritoneum. Dissemination to the central nervous system, particularly leptomeningeal involvement, is extremely rare in this type of tumor. Furthermore, it is usually a late affectation, which in many cases is diagnosed post-mortem.

\section{Case report}

67-year-old woman diagnosed in July 2018 with stage IV pancreatic adenocarcinoma, with a neoformation at the head of the pancreas/ uncinated process and adenopathic conglomerate in liver hilum, retroperitoneal adenopathies and a single retroesophageal adenopathy.

Immediately after diagnosis, the patient began therapy with FOLFIRINOX chemotherapy. With the first cycle of chemotherapy, hospital admission is required for grade IV hematological toxicity and associated sepsis, and it was decided to change chemotherapy regimen to Gemcitabine-albumin bound paclitaxel. Five months after diagnosis and after six cycles of chemotherapy, a tomography scan (CT) is performed to re- evaluate the partial response.

At two months there is a worsening of the analysis, with elevation of serum tumor marker, CEA, and deterioration of the liver profile. All these data point to a progression of the disease, so CT scan is requested describing stability of the disease at the abdominal level but with the appearance at the pulmonary level of pseudonodular formations and an increase in the size of the paratracheal adenopathies. To confirm tumor progression at the lung level, histological samples of subcarinal adenopathy are taken by endoscopic ultrasound (EUS) and paratracheal adenopathy by endobronchial ultrasound (EBUS), both being positive for adenocarcinoma of pancreato- biliary origin.

At this time, a second-line of chemotherapy with FOLFOX regimen is stated. At four months partial radiological improvement and decrease in CEA is observed. At 16 months after diagnosis, and after 13 cycles of FOLFOX, the patient begins with clinical deterioration and neurological symptomatology consisting of instability of the gait and dizziness with turning of objects accompanied by nausea and vomiting. She does not present fever or other infectious signs. At this time, we performed reevaluation of the disease, being stable at the abdominal level and the appearance of a nodule in the middle lobe, and a first brain CT scan without contrast without findings.

Given the persistence of the clinical picture, we decided to admit and repeat CT with contrast iv, which describes findings compatible with leptomeningeal involvement in posterior cranial fossa (Figure 1), these findings being subsequently confirmed by brain magnetic resonance imaging (MRI) (Figure 2).

After this, the patient begins to deteriorate clinically, so we increase the dose of corticoid, experiencing slight improvement, and we perform lumbar puncture, obtaining a sample cerebrospinal fluid (CSF).

In the results of the biochemical analysis (Table 1) of the CSF, significant protein elevation, hypoglycorrhachia and scarce leukocytes stand out. The cytology is negative for tumour cells.

48 hours after the tests were performed, the patient deteriorated sharply on a neurological level, with notable psychomotor agitation and confusion. Palliative sedation was started, and the patient died after 5 days.

${ }^{\star}$ Correspondence to: Garcia Escobar, Medical Oncology Department. General Universitary Hospital of Ciudad Real, Spain, E-mail: naxto@hotmail.com

Key words: pancreatic cancer, leptomeningeal carcinomatosis, leptomeningeal disease

Received: February 08, 2020; Accepted: February 26, 2020; Published: March 02,2020 


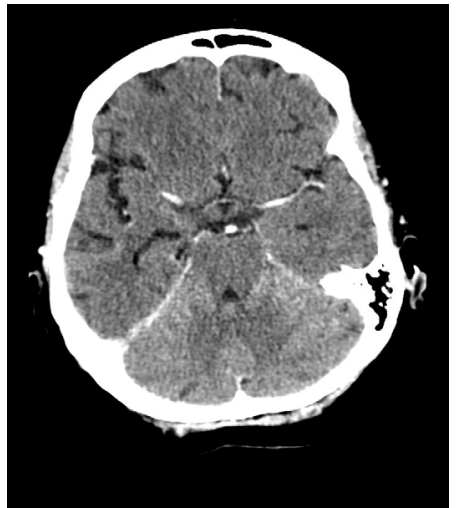

Figure 1. Brain CT showings linear contrast uptake at the posterior fossa level

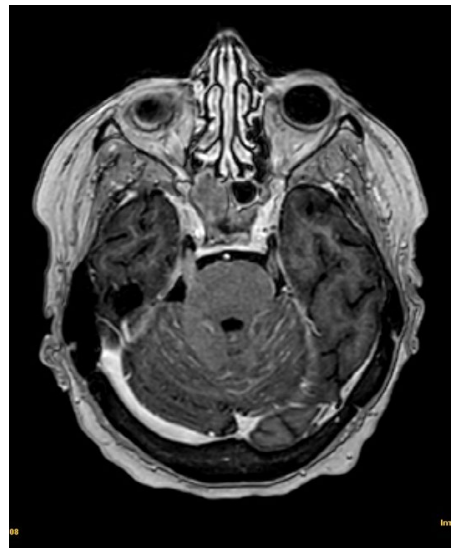

Figure 2. Brain MRI findings: Linear contrast capture and signal alteration at the level of the fourth ventricle and multiple cerebellar folia

Table 1. CSF analysis results

\begin{tabular}{|c|c|}
\hline Appearance & Grossly bloody \\
\hline Red blood cells & $70 / \mathrm{mm}^{3}$ \\
\hline White blood cells & $3 / \mathrm{mm}^{3}$ \\
\hline Glucose & $10 \mathrm{mg} / \mathrm{dl}$ \\
\hline Protein & $221.9 \mathrm{mg} / \mathrm{dl}$ \\
\hline Chloride in CSF & $119.5 \mathrm{mEq} / \mathrm{L}$ \\
\hline Adenosine deaminase level & $10.2 \mathrm{UI} / \mathrm{L}$ \\
\hline Gram Stain & Negative \\
\hline
\end{tabular}

\section{Discussion}

Leptomeningeal carcinomatosis occurs in approximately $3-8 \%$ of patients with solid tumors [2], with the number of cases increasing after autopsy. The most frequently associated primary tumors are lung and breast cancer. Tumors of the gastrointestinal system are very rare, with a limited number of cases reported in the literature.

Leptomeningeal involvement presents with non-specific symptoms such as headache, nausea, vomiting, signs of meningeal irritation or other signs of neurological deficit.
Because of this, it is difficult to diagnose, and requires a high rate of suspicion based on the patient's medical history and symptoms. The definitive diagnosis is made with the cytology of the CSF, however the sensitivity of a single puncture is approximately $50 \%$, which can increase up to $90 \%$ with repeat punctures $[3,4]$. Some of the data that could help the diagnosis would include high opening pressure when performing lumbar puncture, increased proteins and decreased glucose levels in CSF.

Another tool that can support the diagnosis in the presence of a negative CSF cytology is brain MRI with contrast. Using this imaging technique we can observe diffuse meningeal enhancement with nodular deposits in the meninges $[5,6]$.

In the face of clinical suspicion and given that the sensitivity of the cytology is limited, imaging has become the initial, and often the only, diagnostic tool with sensitivity between $76-87 \%[4,6]$.

Treatment of leptomeningeal carcinomatosis is palliative, aiming to improve symptoms and prolong survival, which is median 4-6 weeks without treatment and 3 months with treatment [4-6].

There is no standardized treatment, and depending on the patient's baseline situation, a choice can be made between supportive care, intrathecal chemotherapy and/or radiotherapy.

There is little experience among the intrathecal chemotherapy regimen used, since the available data are based on isolated cases. Among the schemes that have been used are the combination of intrathecal methotrexate, thiotepa and cytarabine liposomal [5], intrathecal hydrocortisone, methotrexate and cytarabine with systemic gemcitabine [7], all with limited effectiveness.

There are published case data on intrathecal topotecan in combination with metrotrexate, and systemic treatment with capecitabine, irinotecan and bevacizumab, which appears to increase survival and good quality of life [2].

In our exposed case, taking into account the poor general condition of the patient, treatment was chosen for symptom control.

\section{References}

1. https://seom.org/info-sobre-el-cancer/pancreas?start=3.

2. Johnson WR, Theeler BJ, Van Echo D, Young P, Kwok M (2018) Treatment of leptomeningeal carcinomatosis in a patient with metastatic pancreatic cancer: A case report and review of the literature. Case Rep Oncol 11: 281-288. [Crossref]

3. Trinh V, Medina-Flores R, Chohan M (2016) Leptomeningeal carcinomatosis as primary manifestation of pancreatic cancer. J Clin Neurosci 30: 124-127. [Crossref]

4. Jindal S, Adithya G, Madaan V, Gupta R, Tandon V, et al. (2018) A rare case of carcinoma pancreas with meningeal metastasis. Apollo Med 15: 223-225.

5. Yoo IK, Lee HS, Kim CD, Chun HJ, Jeen YT, et al. (2015) Rare case of pancreatic cancer with leptomeningeal carcinomatosis. World J Gastroenterol 21: 1020-1023. [Crossref]

6. Clarke JL, Perez HR, Jacks LM, Panageas KS, Deangelis LM (2010) Leptomeningeal metastases in the MRI era. Neurology 74: 1449-1454. [Crossref]

7. Minchom A, Chan S, Melia W, Shah R (2010) An unusual case of pancreatic cancer with leptomeningeal infiltration. J Gastrointest Cancer 41: 107-109. [Crossref]

Copyright: $(2020$ Jimenez Duque D. This is an open-access article distributed under the terms of the Creative Commons Attribution License, which permits unrestricted use, distribution, and reproduction in any medium, provided the original author and source are credited. 